\title{
Emergence delirium in children is not related to intraoperative burst suppression - prospective, observational electrography study
}

Susanne Koch ${ }^{1,4^{*}}$ (D, Anna-Maria Stegherr ${ }^{1}$, Leopold Rupp ${ }^{1}$, Jochen Kruppa ${ }^{2,4}$, Christine Prager ${ }^{3}$, Sylvia Kramer ${ }^{1}$, Astrid Fahlenkamp ${ }^{1}$ and Claudia Spies ${ }^{1}$

\begin{abstract}
Background: Emergence-delirium is the most frequent brain dysfunction in children recovering from general anaesthesia, though the pathophysiological background remains unclear. The presented study analysed an association between emergence delirium and intraoperative Burst Suppression activity in the electroencephalogram, a period of very deep hypnosis during general anaesthesia.
\end{abstract}

Methods: In this prospective, observational cohort study at the Charité - university hospital in Berlin / Germany children aged 0.5 to 8 years, undergoing planned surgery, were included between September 2015 and February 2017. Intraoperative bi-frontal electroencephalograms were recorded. Occurrence and duration of Burst Suppression periods were visually analysed. Emergence delirium was assessed using the Pediatric Assessment of Emergence Delirium Score.

Results: From 97 children being analysed within this study, 40 children developed emergence delirium, and 57 children did not. Overall 52\% of the children displayed intraoperative Burst Suppression periods; however, occurrence and duration of Burst Suppression (Emergence delirium group 55\% / $261+462$ s vs. Non-emergence delirium group 49\% / $318+531$ s) did not differ significantly between both groups.

Conclusions: Our data reveal no correlation between the occurrence and duration of intraoperative Burst Suppression activity and the incidence of emergence delirium. Burst Suppression occurrence is frequent; however, it does not seem to have an unfavourable impact on cerebral function at emergence from general anaesthesia in children.

Trail registration: NCT02481999, June 25, 2015.

Keywords: Burst suppression, Paediatrics: pre-operative anxiety, EEG: high dose opiates

\section{Background}

Postoperative delirium is the most frequent brain dysfunction in patients recovering from general anaesthesia,

* Correspondence: susanne.koch@charite.de

Susanne Koch presented the data on the European Congress of Anaesthesiology 2018 in Copenhagen as Poster (Abstract Reference 2312). ${ }^{1}$ Department of Anaesthesiology and Intensive Care Medicine, Charité-Universitätsmedizin Berlin, Campus Virchow-Klinikum and Campus Charité Mitte, Augustenburger Platz 1, 13353 Berlin, Germany

${ }^{4}$ Berlin Institute of Health (BIH), Anna-Louisa-Karsch 2, 10178 Berlin, Germany Full list of author information is available at the end of the article mainly seen in preschool children as well as in elderly patients $[1,2]$.

In preschool children, postoperative delirium occurs during emergence from anaesthesia during the stay in the post-anaesthesiological care unit (emergence delirium) and presents with acute disorientation, crying, agitation and missing response to the surroundings [3]. Emergence delirium (ED) in children is mostly self-limited and has a benign course. However, it increases the risk of self-injury and induces stress to the medical staff as well as to the caregivers. The implications on

(c) The Author(s). 2019 Open Access This article is distributed under the terms of the Creative Commons Attribution 4.0 International License (http://creativecommons.org/licenses/by/4.0/), which permits unrestricted use, distribution, and 
long-term outcomes especially regarding cognitive function in children is still under discussion.

Pediatric anaesthesiologists are concerned, since it has been proposed that anaesthetic agents may be neurotoxic to the developing brain $[4,5]$.

Elevated concentrations of the anaesthetic agent sevoflurane cause an increase of epileptiform discharges during anaesthesia induction in children [6]. In a former EEG analysis - focusing on the period of anaesthesia induction in the same patient cohort that is presented here we have shown that the occurrence of interictal, epileptiform discharges is positively related to the development of ED [7]. In a study from Martin and colleagues analysing multichannel EEG characteristics in 12 children, from whom 5 developed ED, ED was associated with arousal from an indeterminate state (low voltage / fast frequency EEG activity) and an increased frontal cortical functional connectivity [8]. All these EEG characteristics - interictal, epileptiform discharges, indeterminate state, and increased frontal cortical functional connectivity - are related to a cortical state of hyperexcitability.

On the other hand, elevated concentrations of anaesthetic agents will induce a deeper levels of sedation and periods of Burst Suppression in the EEG during general anaesthesia. It has been proven that periods of Burst Suppression and a low index level of anaesthesia trigger postoperative delirium in elderly patients [9-12]. In children, however, two studies analyzing the index level of anaesthesia and its relationship with the occurrence of ED did not find this correlation [13, 14]. A study focusing on intraoperative Burst Suppression periods and the correlation with ED in children is still lacking.

The aim of this study is to analyse if the occurrence and duration of Burst Suppression patterns during general anaesthesia are related to the development of ED in children.

\section{Methods}

This prospective, observational cohort study (NCT02481999) was approved by our local ethics committee on 12th March 2015 (Ethics committee Charité, University Medicine of Berlin / Chairperson Prof. Dr. R. Seeland / EA2/027/15). Written informed consent of either parents or legal proxies was obtained according to the Declaration of Helsinki. We included children aged 0.5-8 years, undergoing elective surgery with a planned duration of $>60$ min at our University hospital - Charité / Berlin. Between September 8, 2015 and February 22, 2017 parents or legal proxies of the child were approached by study staff members during stay in the preoperative evaluation center on the day prior to surgery. Exclusion criteria comprised any history of neurological or psychiatric diseases, any signs of delayed development in the child, isolation required because of multiresistant bacteria, inability of the parents to speak, read or understand the German language, as well as concurrent enrollment in another study.

The a priori primary outcome was ED assessed during stay in the recovery room with the Pediatric Assessment of Emergence Delirium (PAED) Score [15]. Values of at least 10 were considered as ED.

Secondary outcomes related to frontal EEG recordings were depth of anaesthesia and burst suppression duration.

Oral premedication with midazolam was administered in all children. Children were anaesthetised with either propofol or sevoflurane as decided by the anaesthesiologist in charge. Standard monitoring included non-invasive blood pressure, electrocardiogram and pulse oximetry. Patients received i.v. propofol (assessed as mg kg-1 body weight) or mask induction / maintenance with sevoflurane (assessed as endtidal concentrations et Vol\%). Dosage of sevoflurane and propofol was given according to clinical needs and chosen by the anaesthesiologist in charge. Remifentanil was administered as analgesic agent during the induction period in all children, according to clinical needs. If muscle relaxation was needed, cis-atracurium was administered adapted to body weight. Before the end of surgery children received metamizol or paracetamol and / or piritramid for analgesia. Some children also received regional anaesthesia. The complete anaesthetic procedure and medication were outside of the scope of this study.

\section{ED assessment}

ED was assessed according to the PAED Score. From admission to the post-anaesthesiological care unit until discharge the PAED score was determined $1 \mathrm{~min}$ after extubation, at arrival in the recovery room, $5 \mathrm{~min}, 10$ min, $15 \mathrm{~min}, 30 \mathrm{~min}, 45 \mathrm{~min}$, and $60 \mathrm{~min}$ after arrival and at discharge from the recovery room by a member of the study staff sitting next to the child. Values $>10$ were considered as an emergence delirium [15]. The "Faces Legs Activity Cry Consolability Pain Scale" was used to determine pain events [16]. Richmond Agitation Sedation Scale score was used to assess the level of consciousness [17]. PAED score was only included in the analysis if the Richmond Agitation Sedation Scale score was above -2 and if it was unlikely that pain was triggering agitated behavior. If inadequate behavior during the stay in the recovery room improved after pain-medication these periods of agitated behavior were not classified as ED. If a member of the study team was unable to take the PAED score in the recovery room the child was excluded from further evaluation.

\section{EEG recording and analysis}

Bi-frontal EEGs were obtained with the Narcotrend Monitor (MT Monitor Technik, Bad Bramstedt, Germany). 
The EEG was recorded continuously from baseline before start of anaesthesia until the end of stay in the recovery room. After skin preparation with alcohol four electrodes (Ambu BlueSensor, Bad Nauheim Germany) were placed on the patients' forehead at positions Fz, F7 and F8, with a reference electrode at $\mathrm{Fp} 2$. The impedances were kept below $8 \mathrm{k} \Omega$, differences between electrodes were less than $2 \mathrm{k} \Omega$. During the EEG recording event markers comprising "start of anaesthesia", "intubation", "operation" and "extubation" and "recovery room" were placed. "Start of anaesthesia" was defined as the time-point when the anaesthesiologist began to administer the anaesthetic agent, i.e., either sevoflurane, propofol or a mixture of both. "Intubation" was defined as the time point, when the anaesthesiologist in charge began to intubate the child. "Operation" indicated a time point within $15 \mathrm{~min}$ to 30 min after intubation of stable surgery and anaesthesia, without severe pain events or intraoperative bolus application of propofol. "Extubation" was defined as the time point, at which the anaesthesiologist in charge extubated the child.

Raw EEG data were recorded with a high pass filter of $0.5 \mathrm{~Hz}$ and a low pass filter of $45 \mathrm{~Hz}$, sampling rate was 128 / sec. Visual EEG analysis (EEG viewer software: $50 \mu \mathrm{V}-100 \mu \mathrm{V}$ and $1 \mathrm{~s} / \mathrm{div}$ ) was performed from the time point of "start of anaesthesia" until "extubation". The raw EEG was analysed by an expert (S. K., neurologist with specialization in clinical neurophysiology and EEG) blinded to the ED outcome, the medication patients received and further clinical data. The presence of Burst Suppression periods was validated by a second expert (C.P. pediatric neurologist with specialization in electroencephalography in children). Short periods $(<5 \mathrm{~min}$ within the total anaesthesia procedure) with artifacts (muscle, eyelid, and electricity) were excluded from any further analysis. If persistent artifacts or repeating artifacts were seen, these EEGs were excluded from further analysis.

Burst Suppression periods were assessed by visual inspection of the raw EEG (EEG viewer software: $50 \mu \mathrm{V}$ and $1 \mathrm{~s} /$ div). Burst Suppression segments were included if duration of isoelectric line exceeded $0.5 \mathrm{~s}$. Duration of intraoperative Burst Suppression was calculated from start of the first isoelectric line segment until the end of the last isoelectric line. Isoelectric line is classified as an EEG activity below $5 \mu \mathrm{V}$ [18]. We calculated the duration of the isoelectric line activity by distracting the time duration of the intermittent burst activity. Duration of the isoelectric line is the sum of all periods of isoelectric line present in the raw EEG. Additionally, we calculated the Burst Suppression strength by dividing total isoelectric line duration by Burst Suppression duration. The Burst Suppression strength indicates the fraction of the isoelectric line within the Burst Suppression pattern, a prolonged duration of an isoelectric line will show a
Burst Suppression strength value tending towards 1, which indicates a deeper stage of coma or a deeper level of anaesthesia.

\section{Statistical analysis}

The present study was designed as a prospective, observational study. Statistical analysis was performed using SPSS, version 25, copyright SPSS, Inc., Chicago, IL 60606, USA. Data are presented as means + SD, in case of unbalanced data distribution as medians (IQR 25/75) or as frequencies (\%). For nominal data statistical analysis was performed with the Chi-square test from Pearson. $\mathrm{Nu}$ merical data were analysed using the Mann-Whitney-U test for non-parametric data. To determine the impact of age, anaesthetic medication, Burst Suppression and depth of anaesthesia on the incidence of ED we performed a univariate logistic regression. Odds Ratio with 95\% confidence intervals and corresponding $p$-values were calculated for each risk factor. The Spearman Rho correlation test was used to analyse the correlation between age (in months) and Burst Suppression duration, isoelectric line duration and Burst Suppression strength. Statistical significance was assumed at $p<0.05$.

The statistical analysis plan was made prior to data assessment. To calculate the sample size needed we postulated an ED incidence of $10.5 \%$ (from a pilot study in our department 2013 (NCT02358278), resulting in a $10.5 \%$ incidence of ED in children aged 0-14 years) and an increased risk to develop ED with an increase in depth of anaesthesia and the occurrence of Burst Suppression periods. Four hundred seventy patients were initially planned to be included in this study. An interim analysis was planned (after approximately $1 / 3-1 / 2$ of the total planned sample size) with recalculation of the initial sample size calculation to adopt the study procedure, if the initial assumptions differed strongly. At the interim analysis the ED incidence rate was distinctly higher, which is most likely related to the fact that we mainly included younger patients in our actual study compared to the pilot study 2013 in our department. We re-ran the sample size calculation taking into account the new incidence rate of $41 \%$. Initially, the study was planned for an odds ratio of 1.6 with an ED incidence rate of $10.5 \%$ and a $\mathrm{R} 2$ of the other covariates of 0.2 resulting into a sample size of 470 children with a power of $80 \%$. Considering the new incidence rate of $41 \%$ and without changes of the other parameters we achieve a power of $80 \%$ with 97 children and an odds ratio of 2.1.

\section{Results}

A total of 412 children were screened at the preoperative evaluation center. One hundred eighty-nine children have been included in this prospective observational 
study. The study suffers a high dropout rate based on different reasons. Especially EEG recordings were incomplete because of agitated behaviour in children during anaesthesia induction making it impossible to applicate EEG electrodes before anaesthesia induction without increasing the discomfort or reduce the safety for the young children. Finally, intraoperative EEG data analysis could be performed from "start of anaesthesia" until "extubation" in 97 children (Additional file 1: Figure S1, Flow chart).

Of these 97 children 40 (41\%) children developed Emergence delirium (ED group) and 57 (59\%) did not (Non-ED group). Patients' characteristics are presented in Table 1 . ED patients were significantly younger of age $(P=0.042)$.

$51.5 \%(n=50)$ of all children developed Burst Suppression periods during the anaesthesia procedure, while the remaining $48.5 \%(n=47)$ did not. Burst Suppression started approximately within $\sim 4 \mathrm{~min}$ after "start of anaesthesia" (223 s (IQR 123 to 412), and ended $\sim 11 \mathrm{~min}$ after "start of anaesthesia" (676 s (IQR 417 to 1413)). Burst Suppression duration was $294+502 \mathrm{~s}$ and isoelectric line duration was median $179+375 \mathrm{~s}$.

Occurrence and duration of Burst Suppression, duration of isoelectric line, as well as Burst Suppression strength was not significantly different between ED patients and Non ED patients (Table 2).

Calculation of univariate logistic regression for confounders considered as risk factors triggering ED [age (months), anaesthetic agent given at induction / maintenance (sevoflurane vs. propofol), anaesthesia duration (min), and EEG suppression (occurrence, duration (sec), strength] only age $(P=0.046)$ and anaesthesia duration $(P=0.025)$ showed a significant association (Table 3$)$.

To rule out the risk that younger age has biased our results we recalculated the impact of Burst Suppression duration (sec), isoelectric line duration (sec) and Burst Suppression strength on ED in a pair - wise age matched group (matching rules: age should not differ more than 2 months in children younger than 60 months and not more than 6 months in children being older than 60 month). But again we did not find an impact of Burst Suppression duration, isoelectric line duration and Burst Suppression strength on the risk to develop ED (Additional file 2: Table S1).

Burst suppression periods occurred more frequently in children receiving a mixed induction of anaesthesia with sevoflurane and propofol, compared with children receiving propofol or sevoflurane alone (Burst suppression occurrence: sevoflurane induction 24\%; propofol induction $46 \%$, mixed induction $68 \% ; \mathrm{R}^{2} 0.323, P=0.001$ )

Table 1 Patients' characteristics for children with emergence delirium and children without emergence delirium. Values are median (IQR range), number (propotion) or mean $\pm \mathrm{SD}$

\begin{tabular}{|c|c|c|c|}
\hline & $\begin{array}{l}\text { Non-ED Group } \\
(n=57)\end{array}$ & $\begin{array}{l}\text { ED Group } \\
(n=40)\end{array}$ & $P$ value \\
\hline Age [months] & $58(15.75 / 78)$ & $22(13 / 60)$ & 0.042 \\
\hline Weight (kg) & $17(9.7 / 22)$ & $11(9.3 / 22)$ & 0.142 \\
\hline Height (cm) & $107.5(80 / 131)$ & $86(81.5$ / 119.5) & 0.255 \\
\hline ASA Score (I / II / III) (\%) & $\begin{array}{l}46 / 11 / 0 \\
(81 / 19 / 0)\end{array}$ & $\begin{array}{l}32 / 7 / 1 \\
(80 / 17,5 / 2,5)\end{array}$ & 0.480 \\
\hline Gender male / female (\%) & $\begin{array}{l}35 / 22 \\
(61 / 39)\end{array}$ & $\begin{array}{l}25 / 15 \\
(62,5 / 37,5)\end{array}$ & 0.913 \\
\hline
\end{tabular}

\author{
Cleft-lip-palate; Oral / neck surgery \\ Inguinal hernia / Circumcision / Orchidopexy / Cystoscopy \\ Otorhinolaryngology surgery \\ Intraabdominal surgery / long procedures (> $60 \mathrm{~min}$ ) \\ Limb surgery / short procedures ( $<60 \mathrm{~min}$ ) \\ Midazolam premedication ( $\mathrm{mg} / \mathrm{kg}$ body weight) \\ Induction Agent Sevoflurane / Propofol / mixed (\%)
}

Maintance Agent Sevoflurane / Propofol / mixed (\%)
Procedures (\%)

$6(11)$
$15(26)$
$7(12)$
$14(25)$
$15(26)$
$0.64 \pm 0.17$
$12 / 23 / 22$
$(21 / 41 / 39)$
$38 / 18 / 1$
$(67 / 31 / 2)$
$102+71$

$13(33)$

8 (20)

$4(10)$

9 (23)

$6(15)$

$0.67 \pm 0.18$

$9 / 13 / 18$

$(22,5 / 32,5 / 45)$

$27 / 13 / 0$

$(67,5 / 32,5 / 0)$

$146 \pm 113$
0.180

0.102

0.159 
Table 2 EEG suppression for children with Emergence Delirium and children without emergence delirium. Values are number (propotion) or mean \pm SD

\begin{tabular}{llll}
\hline & $\begin{array}{l}\text { Non-ED Group } \\
(n=57)\end{array}$ & $\begin{array}{l}\text { ED Group } \\
(n=40)\end{array}$ & $P$ value \\
\hline Burst Suppression yes / no (\%) & $\begin{array}{l}28 / 29 \\
(49 / 51)\end{array}$ & $\begin{array}{l}22 / 18 \\
(55 / 45)\end{array}$ & 0.569 \\
Burst Suppression duration (sec) & $318 \pm 531$ & $261 \pm 462$ & 0.984 \\
Isoelectric line duration (sec) & $192 \pm 407$ & $159 \pm 328$ & 0.889 \\
Burst Suppression strength & $0.55 \pm 0.32$ & $.59 \pm 0.31$ & 0.762 \\
\hline
\end{tabular}

Comparing EEG suppression during anaesthesia for children with Emergence delirium (ED group) and children without Emergence delirium (Non ED group). (Chi-square Pearson and Mann-Whitney-U Test). Burst Suppression strength is calculated by dividing isoelectric line duration over Burst Suppression duration.

(Additional file 3: Figure S2). Duration of Burst Suppression, as well as duration of isoelectric line were related to the anaesthetic agents given for induction and maintenance (Additional file 4: Figure S3).

We did not find a correlation between Burst Suppression duration (sec) and isoelectric line duration (sec) and age (months), concentration of midazolam ( $\mathrm{mg} \mathrm{kg}^{-1}$ body weight) premedication or concentration of remifentanil $\left(\mu \mathrm{g} \mathrm{kg} \mathrm{g}^{-1}\right.$ body weight $\mathrm{min}^{-1}$ ) intraoperatively. However, Burst Suppression strength showed a high correlation with age (in months), a tendency to display a longer fraction of isoelectric line within the Burst Suppression pattern in younger children (Spearman-Rho Correlation coefficient $0.528, p<0.0001$; Additional file 5: Table S2 and Additional file 6: Figure S4).

\section{Discussion}

We were able to show that occurrence and duration of Burst Suppression in children aged $0.5-8$ years is not associated with the incidence of ED. Overall, Burst Suppression occurred in about $52 \%$ of all children aged $0.5-8$ years, appearing mainly within the first $\sim 4 \mathrm{~min}$ after application of the anaesthetic agents at start of anaesthesia.
Delirium is the most frequent brain dysfunction seen after anaesthesia procedures, mainly occurring in elderly patients and preschool children [19]. In elderly patients it has been shown that periods of Burst Suppression and a deep index level of anaesthesia are related to postoperative delirium [9-12], however, in two studies analyzing the index level of anaesthesia in children a relationship with the occurrence of ED was not found [13, 14].

Faulk and colleagues examined 400 children aged 112 years scheduled for dental procedures. Deep hypnosis was defined as a level of BIS index reading of less than " 45 ". They did not find a correlation between deep hypnosis and the occurrence of ED [13].

Frederick and colleagues undertook a randomized controlled trial including 40 children aged 2-8 years, randomized in a low-normal group (BIS index values 40-45) versus high-normal group (BIS index values 5560). They found no significant effect of deep versus light anaesthesia on the incidence of ED [14].

In both studies the underlying hypothesis that deep index levels of anaesthesia in children might be related to ED, was not confirmed. This is in line with our data, as we found that the occurrence and duration of Burst Suppression activity was not related to ED in children. Moreover, we extended this conclusion, since our results are based on raw EEG data analysis, which is known to be more reliable compared to Burst Suppression ratios indicated within the processed EEG [20]. Burst suppression segments in the EEG are characterized by an isoelectric line interrupted by high-voltage EEG bursts, indicating a very deep state of unconsciousness with a marked reduction in brain metabolism [21]. The occurrence of Burst Suppression during general anaesthesia in elderly patients is positively correlated to the incidence of postoperative delirium, hence one might propose that elderly patients struggle to restore the preoperative brain metabolism level. In contrast, in children neuronal hyperexcitability - as seen by occurrence of interictal, epileptiform discharges during anaesthesia induction or

Table 3 Confounders considered risk factors for emergence delirium in children

\begin{tabular}{lllll}
\hline Confounders & Odds ratio & $95 \%$ Cl lower limit & $95 \%$ Cl upper limit & $P$ Value \\
\hline Age (months) & 0.986 & 0.973 & 1.000 & 0.046 \\
Anaesthesia Induction (Sevoflurane / Propofol / mix of both) & 0.691 & 0.275 & 1.737 & 2.420 \\
Anaesthesia Maintenance (Sevoflurane / Propofol) & 0.699 & 0.427 & 32.867 \\
Concentration of Midazolam (mg/kg body weight) & 2.886 & 0.253 & 798.215 \\
Concentration of Remifentanil ( $\mu \mathrm{g} / \mathrm{kg}$ body weight / min) & 0.465 & 0 & 1.010 & 0.971 \\
Anaesthesia duration (min) & 1.005 & 1.001 & 2.848 & 0.393 \\
Burst suppression occurrence (yes / no) & 1.266 & 0.563 & 1.001 & 0.025 \\
Burst suppression duration (sec) & 1 & 0.999 & 1.122 & 0.569 \\
Burst Suppression strength & 0.9 & 0.721 & 0.349 \\
\hline
\end{tabular}

Univariate logistic regression accounting for confounders considered risk factors triggering emergence delirium after general anaesthesia in children. Only age (months) and anaesthesia duration ( $\mathrm{min}$ ) showed a significant association 
increased frontal connectivity during emergence of general anaesthesia - has been related to ED [7, 8]. These striking differences in EEG activity related to delirium in children compared with elderly patients extends into its clinical presentation, where delirious children present with crying, agitation, disorientation and altered response to their surroundings, whereas elderly patients present primarily with a hypoactive form of delirium.

Additionally, we found that preschool children display Burst Suppression periods to a lower extent during general anaesthesia procedures (52\%) compared with older patients (aged $62+14$ years; 89\%), however younger adults seem to have the lowest risk (below 25\%) to show Burst Suppression activity during general anaesthesia $[12,22]$.

\section{Limitations}

In our study children receiving a mask induction with sevoflurane were significantly younger compared with children receiving an i.v. induction with propofol, due to standard operating procedure in our clinic to try to avoid children's discomfort during anaesthesia induction. Since younger age as well as sevoflurane anaesthesia have been described to cause ED, this may have biased our results. However, the Non-ED versus ED-group did not show a significant difference between anaesthetic agent used for induction or maintenance and an agematched sub-group analysis did also not reveal a correlation between Burst Suppression duration and ED.

\section{Conclusion}

Intraoperative Burst Suppression activity in the EEG is not associated with ED in young children. Burst Suppression activity is a characteristic EEG feature of a pathological, profoundly inactivated brain but despite its pathological character this does not render to the occurrence of ED in children.

\section{Additional files}

Additional file 1 : Figure S1 Flow diagram. Showing the study flow chart. (DOCX $38 \mathrm{~kb}$ )

Additional file $\mathbf{2}$ : Table S1 Age-matched subgroup analysis. The table presents in an age-matched subgroup analysis Burst Suppression duration, isoelectric line duration and Burst Suppression strength for children with ED versus Non ED group, to rule out any influence of age on our primary result. (DOCX $20 \mathrm{~kb}$ )

Additional file 3 : Figure S2 Burst Suppression occurrence related to the anaesthetic agent at induction. Burst suppression periods occurred more frequently in children receiving a mixed induction of anaesthesia with sevoflurane and propofol, compared with children receiving propofol or sevoflurane alone. (DOCX $90 \mathrm{~kb}$ )

Additional file 4 : Figure S3 Duration of Burst Suppression activity in the EEG related to the anaesthetic agent used for induction and maintenance. Box plot diagram showing the differences of Burst Suppression duration related to the anaesthetic agent used for anaesthesia induction and maintenance. (DOCX $29 \mathrm{~kb}$ )
Additional file $\mathbf{5}$ : Table S2 Correlation between Burst Suppression duration, isoelectric line duration, Burst Suppression strength and age (month). Spearman Rho correlation analysis showing no correlation between Burst Suppression duration, isoelectric line duration and age (month), but a significant correlation between age (month) and Burst Suppression strength, indicating that younger children show prolonged periods of isoelectric line within a Burst Suppression pattern. (DOCX 19 $\mathrm{kb})$

Additional file 6 : Figure S4 Burst Suppression strength related to age (month). Scatter polt indicating the tendency to display a longer fraction of isoelectric line within the Burst Suppression pattern in younger children. (DOCX $40 \mathrm{~kb}$ )

\section{Abbreviations}

ED: Emergence delirium; EEG: Electroencephalography / Electroencephalogramm

\section{Acknowledgements}

Martin Krebs M.D. and Evjenia Toubekis M.D., Consultant in the department of Anaesthesiology and operative Intensive Care Medicine, Charité Universitätsmedizin Berlin, Campus Charité Mitte and Virchow-Klinikum, Berlin, Germany. The authors thank Martin Krebs and Evjenia Toubekis, who provided intraoperative care for the study patients. The authors thank Victoria Windmann for language editing our manuscript. We acknowledge support from the German Research Foundation (DFG) and the Open Access Publication Funds of Charité - Universitätsmedizin Berlin.

\section{Authors' contributions}

SKo, SKr and CS conceived and designed the experiments. SKo, AMS and LR performed the experiments. SKo, AMS, LR, JK, CP, SKr and AF analysed the data. SKo and CS contributed to the materials/analysis tools. SKo and JK wrote the paper. All authors read and approved the final manuscript.

\section{Funding}

This work was supported by the Charité-Humboldt University Berlin. Susanne Koch was supported by the Lydia-Rabinowitsch Grant from the Charité-Humboldt University Berlin 2013/2014 to design and set up the study. Further support was provided from institutional and departmental sources for data collection, analysis and interpretation of the study data and for writing the manuscript.

\section{Availability of data and materials}

The datasets used and/or analysed during the current study are available from the corresponding author on reasonable request.

\section{Ethics approval and consent to participate}

This prospective, observational cohort study (NCT02481999) was approved by our local ethics committee on 12 March 2015 (Ethik committee Charité, University Medicine of Berlin / Chairperson Prof. Dr. R. Seeland / EA2/027/15). Written informed consent of either parents or legal proxies was obtained according to the Declaration of Helsinki.

\section{Consent for publication}

Not applicable.

\section{Competing interests}

Claudia Spies received material support by Narcotrend-Gruppe, MT Monitortechnik GmbH, Maienbaß 27, 24576 Bad Bramstedt. The authors declare no further competing interests.

\section{Author details}

${ }^{1}$ Department of Anaesthesiology and Intensive Care Medicine, Charité-Universitätsmedizin Berlin, Campus Virchow-Klinikum and Campus Charité Mitte, Augustenburger Platz 1, 13353 Berlin, Germany.

${ }^{2}$ Charité-Universitätsmedizin Berlinlnstitute of Biometry and Clinical

Epidemiology, Campus Charité Mitte, Charitéplatz 1, 10117 Berlin, Germany. ${ }^{3}$ Department of Paediatria and Neurology, Charité-Universitätsmedizin Berlin, Campus Virchow-Klinikum and Campus Charité Mitte, Augustenburger Platz

1, 13353 Berlin, Germany. ${ }^{4}$ Berlin Institute of Health (BIH), Anna-Louisa-Karsch 2, 10178 Berlin, Germany. 
Received: 22 March 2019 Accepted: 31 July 2019

Published online: 08 August 2019

\section{References}

1. Dahmani S, Delivet H, Hilly J. Emergence delirium in children: an update. Curr Opin Anaesthesiol. 2014;27:309-15.

2. Inouye SK, Westendorp RG, Saczynski JS. Delirium in elderly people. Lancet. 2014;383:911-22.

3. Somaini M, Engelhardt T, Fumagalli $R$, Ingelmo PM. Emergence delirium or pain after anaesthesia--how to distinguish between the two in young children: a retrospective analysis of observational studies. $\mathrm{Br}$ J Anaesth. 2016;116:377-83

4. Jevtovic-Todorovic V. Functional implications of an early exposure to general anesthesia: are we changing the behavior of our children? Mol Neurobiol. 2013:48:288-93.

5. Berndt N, Rosner J, Haq RU, et al. Possible neurotoxicity of the anesthetic propofol: evidence for the inhibition of complex II of the respiratory chain in area CA3 of rat hippocampal slices. Arch Toxicol. 2018;92:3191-205.

6. Kreuzer I, Osthaus WA, Schultz A, Schultz B. Influence of the sevoflurane concentration on the occurrence of epileptiform EEG patterns. PLoS One. 2014;9:e89191.

7. Koch S, Rupp L, Prager C, Wernecke KD, Kramer S, Fahlenkamp A, Spies C. Emergence delirium in children is related to epileptiform discharges during anaesthesia induction: an observational study. Eur J Anaesthesiol. 2018;35:929-36

8. Martin JC, Liley DT, Harvey AS, Kuhlmann L, Sleigh JW, Davidson AJ. Alterations in the functional connectivity of frontal lobe networks preceding emergence delirium in children. Anesthesiology. 2014;121:740-52.

9. Radtke FM, Franck M, Lendner J, Kruger S, Wernecke KD, Spies CD. Monitoring depth of anaesthesia in a randomized trial decreases the rate of postoperative delirium but not postoperative cognitive dysfunction. $\mathrm{Br}$ J Anaesth. 2013;110:198-105

10. Chan MT, Cheng BC, Lee TM, Gin T, Group CT. BIS-guided anesthesia decreases postoperative delirium and cognitive decline. J Neurosurg Anesthesiol. 2013;25:33-42

11. Whitlock EL, Torres BA, Lin N, et al. Postoperative delirium in a substudy of cardiothoracic surgical patients in the BAG-RECALL clinical trial. Anesth Analg. 2014;118:809-17.

12. Fritz BA, Kalarickal PL, Maybrier HR, et al. Intraoperative electroencephalogram suppression predicts postoperative delirium. Anesth Analg. 2016;122:234-42.

13. Faulk DJ, Twite MD, Zuk J, Pan Z, Wallen B, Friesen RH. Hypnotic depth and the incidence of emergence agitation and negative postoperative behavioral changes. Paediatr Anaesth. 2010;20:72-81.

14. Frederick HJ, Wofford K, de Lisle Dear G, Schulman SR. A randomized controlled trial to determine the effect of depth of anesthesia on emergence agitation in children. Anesth Analg. 2016;122:1141-6.

15. Sikich N, Lerman J. Development and psychometric evaluation of the pediatric anesthesia emergence delirium scale. Anesthesiology. 2004;100:1138-45.

16. Merkel SI, Voepel-Lewis T, Shayevitz JR, Malviya S. The FLACC: a behavioral scale for scoring postoperative pain in young children. Pediatr Nurs J. 1997:23:293-7.

17. Kerson AG, DeMaria R, Mauer E, Joyce C, Gerber LM, Greenwald BM, et al. Validity of the Richmond agitation-sedation scale (RASS) in critically ill children. J Intensive Care. 2016:4:65

18. Zschocke S. EEG bei diffusen Erkrankungen des Gehirns. In: Hansen H-C, editor. Klinische Elektroenzephalographie. Berlin, Heidelberg, New York: Springer-Verlag; 2011. p. 314-48.

19. Costi D, Cyna AM, Ahmed S, et al. Effects of sevoflurane versus other general anaesthesia on emergence agitation in children. Cochrane Database Syst Rev. 2014. https://doi.org/10.1002/14651858.CD007084.

20. Muhlhofer WG, Zak R, Kamal T, et al. Burst-suppression ratio underestimates absoulte duration of electroencephalogram suppression compared with visual analysis of intraoperative electroencephalogram. Br J Anaesth. 2017;118:755-61.

21. Brown EN, Lydic R, Schiff ND. General anesthesia, sleep, and coma. N Engl J Med. 2010:363:2638-50.

22. Purdon PL, Pavone KJ, Akeju O, Smith AC, Sampson AL, Lee J, et al. The ageing brain: age-dependent changes in the electroencephalogram during propofol and sevoflurane general anaesthesia. Br J Anaesth. 2015;115:i46-57.

\section{Publisher's Note}

Springer Nature remains neutral with regard to jurisdictional claims in published maps and institutional affiliations.

\section{Ready to submit your research? Choose BMC and benefit from:}

- fast, convenient online submission

- thorough peer review by experienced researchers in your field

- rapid publication on acceptance

- support for research data, including large and complex data types

- gold Open Access which fosters wider collaboration and increased citations

- maximum visibility for your research: over $100 \mathrm{M}$ website views per year

At BMC, research is always in progress.

Learn more biomedcentral.com/submissions 